\title{
Tuberculous Spondylitis (Pott's Disease) with Bilateral Pleural Effusion
}

\author{
Yusuke OкuYama, Yusuke NaKaоKA*, Kunihiko Kıмото** and Kotaro Ozasa
}

\begin{abstract}
We report a case of tuberculous spondylitis (Pott's disease) with bilateral pleural effusion in a 25-year-old male patient. Left-pleural effusion was observed on admission. The initial diagnosis was tuberculous pleuritis. However, during anti-tuberculosis chemotherapy, back pain and rightpleural effusion appeared. by further examination, we diagnosed an active tuberculous spondylitis of the 11th and 12th thoracic vertebrae possibly by the dissemination of Mycobacterium tuberculosis through blood circulation or lymphatic circulation. In cases in which anti-tuberculosis chemotherapy is not so effective, the possibility of the rarely-appearing extra-pulmonary manifestation of tuberculous spondylitis must be considered.
\end{abstract}

(Internal Medicine 35: 883-885, 1996)

Key words: Pott's disease, bilateral pleuritis

\section{Introduction}

Tuberculosis is a systemic disease with widespread clinical manifestations. In Japan, the mortality rate, incidence, and prevalence of tuberculosis have decreased remarkably. However, tuberculosis manifests itself in extra-pulmonary organs. One of the most frequent locations is the spine. The symptoms of tuberculous spondylitis are not specific, and the course is creeping, which makes an early diagnosis difficult. Here, we report a patient with tuberculous spondylitis without a past history of tuberculosis or other debilitating diseases such as malignancy, alcoholism, diabetes, and immunodeficiency. We summarize the present epidemiology and clinical diagnosis of tuberculous spondylitis, and discuss the etiopathogenesis of the present patient.

\section{Case Report}

A 25-year-old male was referred to the hospital on October 2, 1990, complaining of continued fever for 10 days. On admission he had low grade fever and productive cough. He had no significant past history or family history. The physical examinations showed blood pressure of $110 / 52 \mathrm{mmHg}$, regular pulse at $78 / \mathrm{min}$, and body temperature of $37.3^{\circ} \mathrm{C}$. Superficial lymph nodes were not palpable. The chest examination revealed that the breath sound was decreased over the left poste- rior base. However, no rale was heard. The initial chest X-ray showed massive pleural effusion on the left side (Fig. 1). The initial blood count showed a hematocrit of $41.6 \%$, leukocyte count of 6,600 with $9 \%$ of stab and $62 \%$ of segment forms, $25 \%$ of lymphocytes and $4 \%$ of monocytes. Laboratory data included an erythrocyte sedimentation rate (ESR) of $28 \mathrm{~mm} / \mathrm{h}$ and C-reactive protein (CRP) of $5.3 \mathrm{mg} / \mathrm{dl}$. Other laboratory data on admission included normal liver function, serum creatinine 0.8 $\mathrm{mg} / \mathrm{dl}$, total protein $7.3 \mathrm{~g} / \mathrm{dl}$, albumin $4.1 \mathrm{~g} / \mathrm{dl}$, and no abnormalities of electrolytes.

\section{For editorial comment, see p 839.}

He had received the BCG vaccine in childhood, and the intradermal tuberculin test on admission was positive with 12 $\times 12 \mathrm{~mm}$ of reddening, without an induration. As revealed by pleurocentesis, the nature of the pleural effusion included the specific gravity of 1.021 , protein of $5.4 \mathrm{~g} / \mathrm{dl}$, and ADA (adenosine deaminase) of $50.2 \mathrm{U} / l$. Cultures from the left side-pleural effusion were negative at 8 weeks. Pathological study detected thickening pleural tissue and the infiltration of lymphocytes. We therefore, made a diagnosis of tuberculous pleuritis and started anti-tuberculosis therapy with isoniazid $300 \mathrm{mg} / \mathrm{day}$, rifampicin $450 \mathrm{mg} / \mathrm{day}$, and streptomycin sulfate $1 \mathrm{~g} /$ day. On October 19, ESR and CRP were decreased to the normal level, and the pleural effusion on the left side was markedly de-

From the Department of Preventive Medicine, Kyoto Prefectural University of Medicine, Kyoto, *the Department of Respiratory Medicine and **the Department of Gastroenterology, Osaka Railway Hospital, Osaka

Received for publication April 2, 1996; Accepted for publication July 24, 1996

Reprint requests should be addressed to Dr. Yusuke Okuyama, the Department of Preventive Medicine, Kyoto Prefectural University of Medicine, Kawaramachi-Hirokoji, Kamigyo-ku, Kyoto 602 


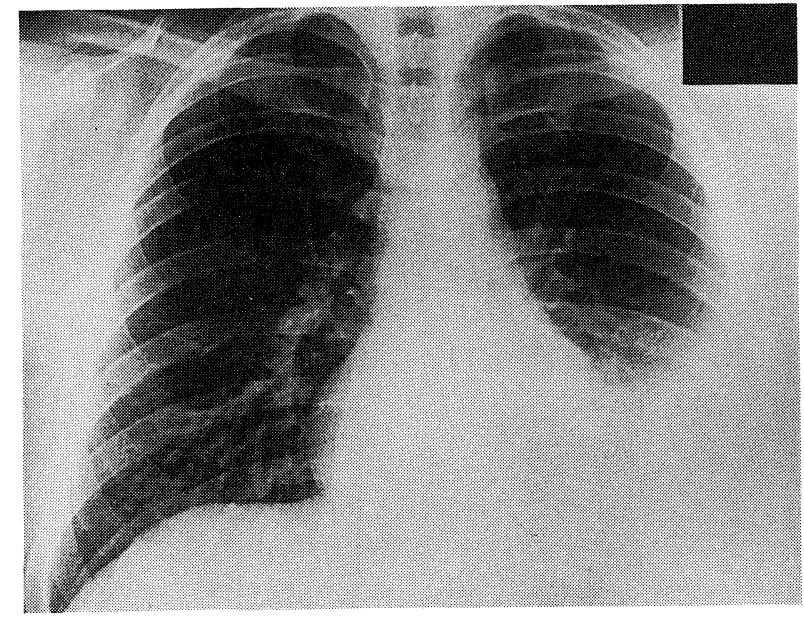

Figure 1. Chest roentgenogram on admission. A massive pleural effusion on the left side was noted.

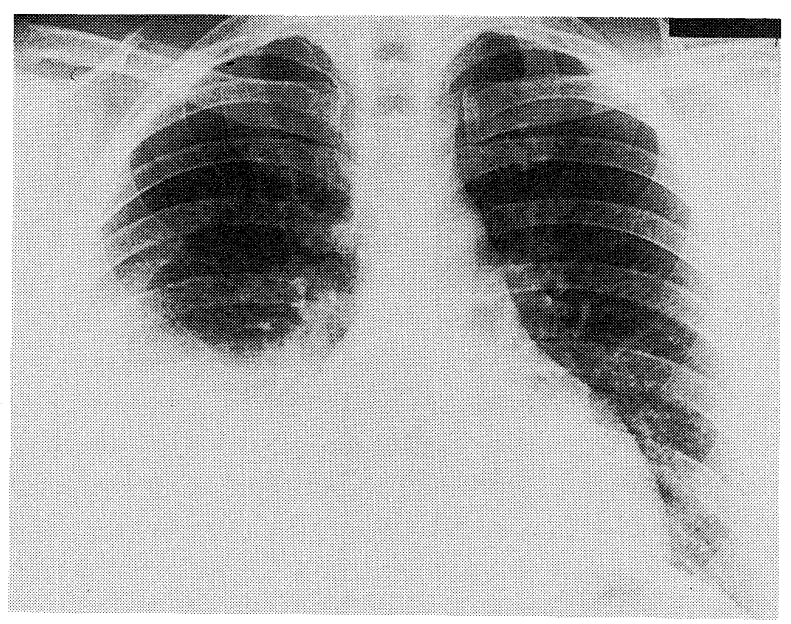

Figure 2. Chest roentgenogram two weeks after the treatment of anti-tuberculous chemotherapy. A massive pleural effusion on the right side was noted.

creased. However, from this time, the patient began to complain of back pain and show remittent fever. On October 26, the ESR and CRP were elevatedto $42 \mathrm{~mm} / \mathrm{h}$ and $5.9 \mathrm{mg} / \mathrm{dl}$, respectively. The chest X-ray showed massive pleural effusion on the right side (Fig. 2). The nature of the right pleural effusion was same as that of the left. Furthermore, by chest and abdominal computed tomography (CT) on November 2, multiple lymph node swelling near the spine and an abscess in the right psoas muscle were pointed out (Fig. 3). Magnetic resonance imaging (MRI) of the spine on November 8 showed the destruction of the 11th and 12th thoracic vertebra (Fig. 4). An abscess biopsy was performed from the right psoas muscle. The histological findings were caseous necrosis with Langhans's giant cells and epithelioid cells (Fig. 5). We thus made the diagnosis of active tuberculous spondylitis of the 11th and 12th thoracic vertebrae. After 8 weeks, cultures confirmed the diagnosis by showing

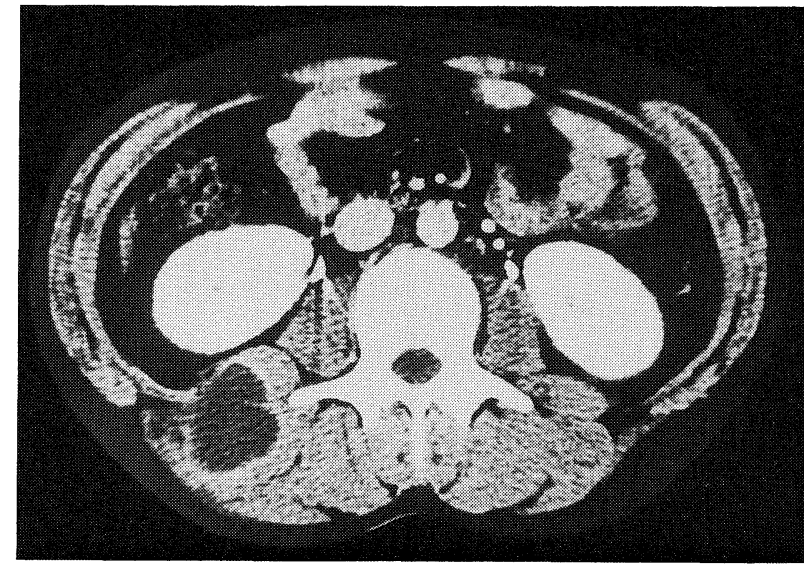

Figure 3. Abdominal computed tomography two weeks after the treatment of anti-tuberculous chemotherapy showed an abscess in the right psoas muscle.

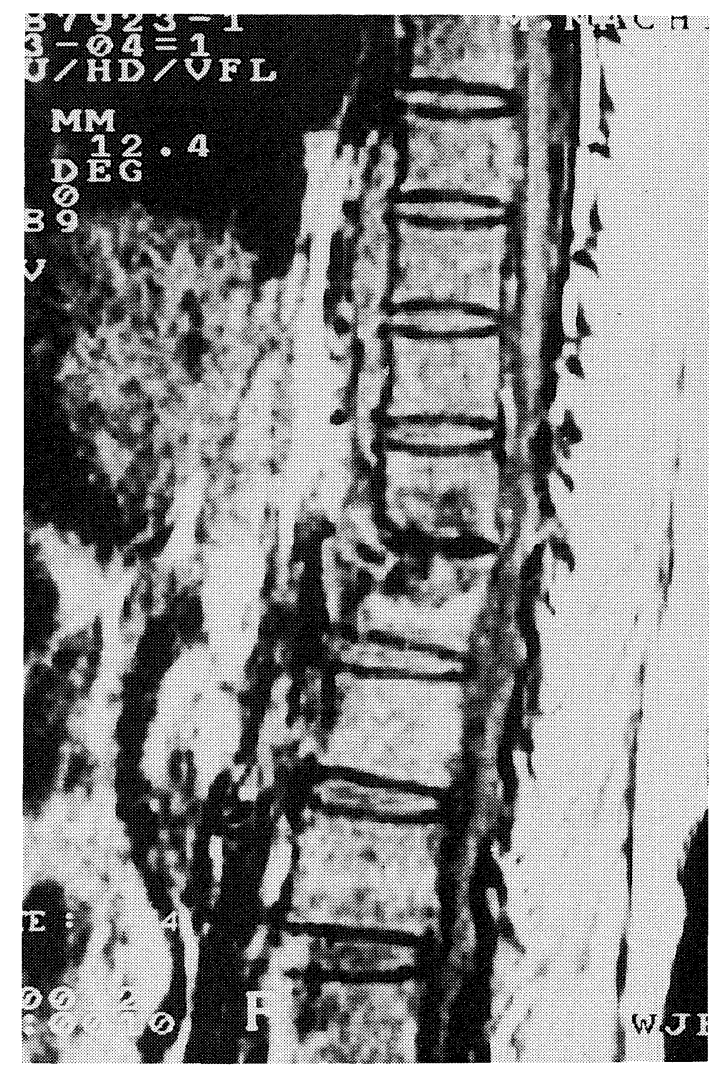

Figure 4. Magnetic resonance imaging showed the destruction of the 11th and 12th thoracic vertebra.

growth of Mycobacterium tuberculosis. The number of colonies was not so many and was one plus by semi-quantitative measurement. The response to anti-tuberculosis chemotherapy was favorable, and the ESR and CRP were markedly decreased. On March 5, 1991, a curative operation for tuberculous spondylitis was performed. The patient underwent resection of the 


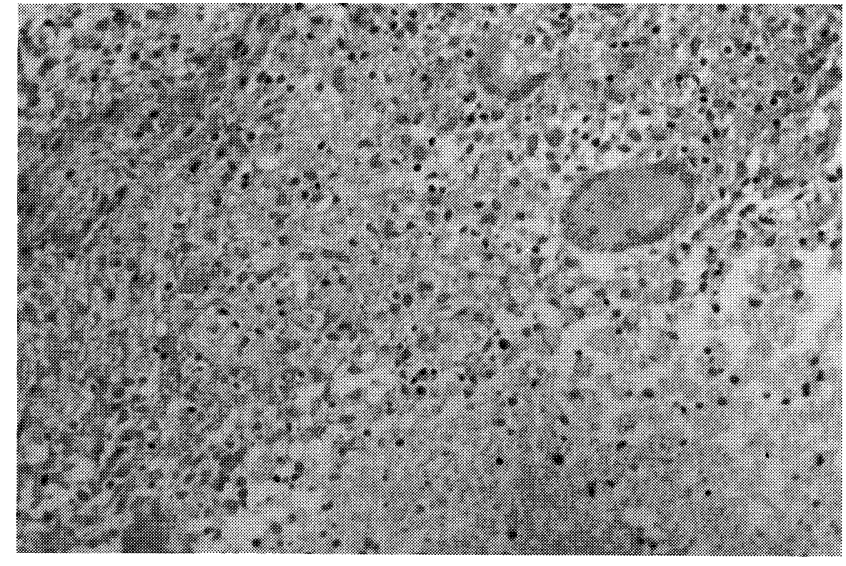

Figure 5. Histological findings of the abscess biopsy from the right psoas muscle (HE stain, $\times 100$ ). Caseous necrosis with Langhan's giant cells and epithelioid cells was revealed.

necrotic bone and anterior spinal fusion using a transplanted bone autograft. His post-operative course was good and he was discharged two months after the curative operation.

\section{Discussion}

It is reported that tuberculosis manifests itself in extrapulmonary organs in about $18 \%$ of the cases (1). Tuberculous spondylitis (Pott's disease) is one of the common complications of Mycobacterium tuberculosis infection except for tuberculous pleuritis. In Japan in 1994, the ratio of patients with tuberculous spondylitis was about $3.3 \%$ of the total number of patients with extra-pulmonary tuberculosis (2). In many industrialized countries, the prevalence of tuberculous spondylitis is gradually increasing. The HIV epidemic has contributed to resurgent tuberculosis. The spread of tuberculosis among the homeless and the expanding immigrant population may also be the another cause (3-5). In the present patient, it was surprising that the severe infection of Mycobacterium tuberculosis had occurred, although the patient had no past history of tuberculosis or other debilitating diseases such as malignancy, alcoholism, diabetes, and immunodeficiency.

Generally, tuberculous spondylitis is a chronic and slowly progressive disease, while pyogenic spondylitis is a more acute disease (6). Tuberculous spondylitis has varied manifestations. The initial subjective symptoms may be relatively nonspecific, such as back pain, vertebral tenderness, fever, chilliness, and body weight loss. In more advanced cases, neurological symptoms and spinal deformity are present. Laboratory data such as an elevated ESR, CRP and positive tuberculin skin test provide some information, but are by themselves not for a firm diagnosis. On the other hand, the radiological findings provide more useful information. Plain X-ray films demonstrate a destructive process of the vertebrae, involvement of the disc space, and spinal deformity (6). The lower thoracic and lumbar spine are most commonly affected (7). From this point of view, CT and MRI provide a great advantage in the differential diagnosis of tuberculous spondylitis. It is reported that MRI has an improved contrast resolution for bone and soft tissues and versatility of direct imaging in multiple planes (8). MRI is more useful to demonstrate paravertebral abscess clearly and more useful in the differential diagnosis of vertebral osteomyelitis and disk space infection than is CT (9). In the present case, the MRI study was informative and useful for the diagnosis.

In the present patient, the left-pleural effusion appeared first, and the right-pleural effusion appeared during the anti-tuberculosis chemotherapy. As for the etiopathogenesis of this phenomenon, it seems that although the primary tuberculosis lesions were not seen, the Mycobacterium tuberculosis was already spread through the blood circulation or lymphatic circulation. In fact, it has been reported that about $50 \%$ of patients with tuberculous spondylitis have chest $\mathrm{X}$-rays that are negative for active or inactive pulmonary tuberculosis (10). Therefore, we suspect that when we started the anti-tuberculosis chemotherapy for tuberculous pleuritis, the tuberculous spondylitis was already latently developed in the spine. In spite of the disappearance of the left-side pleural effusion after chemotherapy, the appearance of the right-side pleural effusion supports this supposition. Another minor possibility to explain the present change for the worse of the tuberculous spondylitis and the appearance of right-side pleural effusion is that the Mycobacterium tuberculosis infection might be resistant to the chemotherapy. However, to our regret, we had not examined the sensitivity tests for anti-tuberculotic drugs; the details are therefore unknown.

In cases in which anti-tuberculosis chemotherapy is not effective, and the ESR increases or remains at a high level, or fever and back pain continue despite chemotherapy, the possibility of the rarely-appearing extra-pulmonary manifestations such as tuberculous spondylitis must be considered, and the diagnosis should be determined as soon as possible.

\section{References}

1) Seaton A, Seaton D, Leitch AG. Crofton \& Douglas's Respiratory Diseases. Blackwell, Oxford, 1989, p. 395.

2) Statistics of T.B. Health Service Bureau, Infectious Disease Control Division, 1994, p. 55.

3) Shafer RW, Kim DS, Weiss JP, Quale JM. Extrapulmonary tuberculosis in patients with human immunodeficiency virus infection. Medicine (Baltimore) 70: 384, 1991.

4) Yamanaka K, Kondo T, and Miyao M. Tuberculosis among the homeless people of Nagoya, Japan. Respir Med 88: 763, 1994.

5) Shanley DJ. Tuberculosis of the spine: imaging features. AJR Am J Roentgenol 164: 659, 1995.

6) William NR and Stuart MG. Tuberculosis. Little, Brown, 1995 p. 623.

7) Scully RE, Mark EJ, McNeely WF, et al. Case records of the Massachusetts General Hospital case 5-1988. N Engl J Med 318: 306, 1988.

8) Kim NH, Lee HM, Suh JS. Magnetic resonance imaging for the diagnosis of tuberculous spondylitis. Spine 19: 2451, 1994.

9) Paushter DM, Modic MT, Masaryk TJ. Magnetic resonance imaging of the spine: applications and limitations. Radiol Clin North Am 23: 551, 1985.

10) Grinkemeyer MD, LaBarre RC, Yaszemski MJ, Klucznik RP, Blatt SP, Drehner DM. A case of Pott's disease in a 20-year-old military dependent. Mil Med 159: 257, 1994. 